\title{
Effects of aerobic exercise training on heart rate variability during wakefulness and sleep and cardiorespiratory responses of young and middle-aged healthy men
}

\footnotetext{
A.M. Catai 1,3 ,

M.P.T. Chacon-Mikahil 3,4 ,

F.S. Martinelli $i^{3,4}$,

V.A.M. Forti ${ }^{4}$, E. Silva ${ }^{1}$,

R. Golfetti ${ }^{4}$, L.E.B. Martins ${ }^{4}$,

J.S. Szrajer ${ }^{4}$, J.S. W anderley,

E.C. Lima-Filho ${ }^{4}$, L.A. Milan²,

J.A. Marin-Neto ${ }^{5}$,

B.C. Maciel ${ }^{5}$

and L. Gallo-Junior ${ }^{5}$
}

\author{
${ }^{1}$ Laboratório de Fisioterapia Cardiovascular, Departamento de Fisioterapia, and \\ ${ }^{2}$ Departamento de Estatística, Universidade Federal de São Carlos, \\ São Carlos, SP, Brasil \\ ${ }^{3}$ Departamento de Fisiologia e Biofísica, Instituto de Biologia, \\ Universidade Estadual de Campinas, Campinas, SP, Brasil \\ ${ }^{4}$ Laboratório de Fisiologia do Exercício, Faculdade de Educação Física, \\ Universidade Estadual de Campinas, Campinas, SP, Brasil \\ ${ }^{5}$ Divisão de Cardiologia, Departamento de Clínica Médica, \\ Hospital das Clínicas, Faculdade de Medicina de Ribeirão Preto, \\ Universidade de São Paulo, Ribeirão Preto, SP, Brasil
}

\section{Correspondence \\ A.M. Catai \\ Laboratório de Fisioterapia \\ Cardiovascular - Núcleo de \\ Pesquisa em Exercício Físico, \\ Departamento de Fisioterapia, \\ UFSCar \\ Rodovia Washington Luís, km 235 \\ 13565-905 São Carlos, SP \\ Brasil \\ E-mail: mcatai@ power.ufscar.br}

Research supported by CAPES, FAPESP (No. 91/4754-9), CNPq (No. 300528/85-0), and FAEP-UNICAMP.

Received July 17, 2001

Accepted April 1, 2002

\section{Abstract}

The purpose of the present study was to evaluate the effects of aerobic physical training (APT) on heart rate variability (HRV) and cardiorespiratory responses at peak condition and ventilatory anaerobic threshold. Ten young $(\mathrm{Y}:$ median $=21$ years $)$ and seven middle-aged $(\mathrm{MA}=$ 53 years) healthy sedentary men were studied. Dynamic exercise tests were performed on a cycloergometer using a continuous ramp protocol (12 to $20 \mathrm{~W} / \mathrm{min}$ ) until exhaustion. A dynamic 24-h electrocardiogram was analyzed by time (TD) (standard deviation of mean R-R intervals) and frequency domain (FD) methods. The power spectral components were expressed as absolute (a) and normalized units (nu) at low (LF) and high (HF) frequencies and as the LF/HF ratio. Control (C) condition: HRV in TD (Y: 108, MA: $96 \mathrm{~ms}$; P<0.05) and FD - LFa, $\mathrm{HFa}$ - was significantly higher in young $\left(1030 ; 2589 \mathrm{~ms}^{2} / \mathrm{Hz}\right)$ than in middle-aged men $\left(357 ; 342 \mathrm{~ms}^{2} / \mathrm{Hz}\right)$ only during sleep $(\mathrm{P}<0.05)$; posttraining effects: resting bradycardia $(\mathrm{P}<0.05)$ in the awake condition in both groups; $\dot{\mathrm{VO}}_{2}$ increased for both groups at anaerobic threshold $(\mathrm{P}<0.05)$, and at peak condition only in young men; HRV in TD and FD (a and nu) was not significantly changed by training in either groups. The vagal predominance during sleep is reduced with aging. The resting bradycardia induced by short-term APT in both age groups suggests that this adaptation is much more related to intrinsic alterations in sinus node than in efferent vagal-sympathetic modulation. Furthermore, the greater alterations in $\mathrm{VO}_{2}$ than in $\mathrm{HRV}$ may be related to short-term APT.

\section{Key words}

- Heart rate variability

- Power spectral density analysis

- Anaerobic threshold

- Aerobic exercise training

- Autonomic nervous system and aging processes in man 


\section{Introduction}

Heart rate variability (HRV) is mainly caused by efferent autonomic modulation of the sinus node. For many years this variable has been expressed only as mean values and standard deviations, i.e., a measure in the time domain representation. However, a noninvasive contribution by each division of autonomic modulation to $\mathrm{HRV}$ is possible when this variable is represented in its frequency domain, i.e., the power spectral density analysis. Today, it is well accepted that under specific experimental conditions the power spectrum is a tool of great value for assessing the neural mechanisms controlling heart rate (HR) (1).

Analysis of HRV in the frequency domain obtained from mathematical processing of the R-R intervals in the electrocardiogram recordings obtained under resting conditions can discriminate two main spectral components: a high frequency one (ranging from 0.15 to $0.40 \mathrm{~Hz}$ ) and a low frequency one (ranging from 0.04 to $0.15 \mathrm{~Hz}$ ), considered to be markers of parasympathetic and sympathetic control, respectively $(1,2)$. However, Skyschally et al. (3) have suggested that low frequency is influenced by both vagal and sympathetic activity.

Measurement of HRV may be useful as a noninvasive method to assess in man the autonomic nervous system modulation under several physiological conditions such as awake and sleeping situations, different body positions, physical training, and also in pathological conditions (1,4-6). Thus, the HRV expressed both in the time and frequency domains is reduced with age $(4,7)$ due to the dominance of the sympathetic over the parasympathetic balance in this particular condition $(4,7)$. This observation is relevant since the reduction of $H R V$ with aging is related to higher cardiovascular morbidity and mortality rates $(7,8)$.

There are conflicting reports in the literature concerning the effects of aerobic train- ing on HRV under resting conditions. While some studies have reported an increase in the magnitude of this variable in the time domain (9), in the frequency domain others have reported absence of modifications (10), and an increase (11) or decrease (12) of sympathovagal balance in the sinus node.

The effect of age on physical working capacity has also been the subject of many studies $(13,14)$ that have shown that maximal aerobic capacity, measured as $\mathrm{VO}_{2}$ max, reaches a maximum value around the age of 30 years and decreases progressively thereafter. Concerning $\mathrm{VO}_{2}$ at the anaerobic threshold, the literature has also shown a decline of this parameter with advancing age (13) and there are studies indicating the occurrence of significant changes in aerobic capacity and autonomic changes in HR after aerobic training in middle-aged subjects $(15,16)$.

On the basis of these considerations, the purpose of the present study was to evaluate the effects of 3-month aerobic physical training on the efferent autonomic cardiac control that modulates the HR response at rest in awake and sleeping conditions and on the oxygen uptake at ventilatory anaerobic threshold and peak conditions during dynamic exercise in young and middle-aged men.

\section{Material and Methods}

\section{Subjects}

Seventeen men volunteered to take part in this study. All of them were in good health based on clinical and physical examination, and laboratory tests that included a standard electrocardiogram (ECG), maximum exercise test (protocol I), chest X-rays, total blood count, urinalysis and clinical biochemical screen tests [glycose, uric acid, total cholesterol and fractions (LDL, HDL and VLDL), and triglycerides]. All subjects had sedentary life-styles and most of them participated only eventually in weekend sport activities. 
None of the subjects studied was taking any type of medication. Two different age groups were compared: young group $(\mathrm{N}=10)$, age range of 19 to 29 years (median $=21$ ), and middle-aged group $(\mathrm{N}=7)$, age range of 50 to 59 years $($ median $=53)$. The subjects were informed about the experimental procedures and all signed an informed consent form to participate in the present study, which was approved by the Ethics Committee of the State University of Campinas. All individuals were evaluated during the same time of day at an experimental room temperature of $23^{\circ} \mathrm{C}$ and relative air humidity between 50 and $60 \%$. Before the day of the experiment the subjects were taken to the experimental room for familiarization with the procedures and the equipment to be used. Each subject had been oriented to avoid caffeinated and alcoholic beverages, to refrain from smoking and not to perform moderate or heavy exercise on the day before the application of protocols I and II or during the 24-h period for the Holter test. On each experimental day, before conducting the programmed protocols, the volunteers were interviewed and examined to confirm the state of good health, the occurrence of a normal night sleep, and to confirm that the control conditions (HR and systemic blood pressure) were in the normal range.

\section{Protocols}

Protocol I. All subjects were studied in the resting condition (supine and seated) and during two dynamic exercise tests in the seated position on a cycloergometer, using a continuous protocol on different days separated by a 2-7-day interval, as follows:

a) Clinical and diagnostic evaluation. The main purpose of this procedure was to include in the study only healthy men, excluding any subject with evidence of silent ischemic heart disease or other pathologic abnormalities of the cardiovascular system. A 12-lead standard ECG recording was ob- tained at rest in the supine position. The exercise protocol consisted of 3-min step power increments of $25 \mathrm{~W}$, with a rotation frequency of $60 \mathrm{rpm}$ maintained throughout the test. The exercise tests ended when the subjects presented one or more of the following conditions: 1) clear evidence of physical exhaustion, 2) reaching the age-predicted maximal HR, and 3) inability to maintain a standard cycling frequency due to muscular fatigue. During the protocol the subjects were monitored using the thoracic CM5 lead. An ECG tracing (CM5, aVF and V2) was obtained during the last $10 \mathrm{~s}$ of each power level. Arterial pressure was measured by the auscultatory method using a mercury sphygmomanometer during the last $15 \mathrm{~s}$ of each power level.

b) Functional capacity evaluation: oxygen uptake test. The subjects performed an oxygen uptake test using a progressive incremental exercise protocol. This protocol consisted of a 3-min warm-up at $4 \mathrm{~W}$ followed by a continuous power increase set at a value of 12 to $20 \mathrm{~W}$ up to physical exhaustion. The choice of the power value increment for each subject, i.e., 12, 15, 17 or $20 \mathrm{~W} / \mathrm{min}$, was based on the responses presented in the previous clinical test described above (protocol I-a). A braked electromagnetic cycloergometer equipped with a microprocessor (model Corival 400, Quinton, Seattle, WA, USA) allowed the precise application of individualized power ramp values. At the peak of effort each subject attributed a rating of perceived exertion based on Borg's scale (17), that varied from 0 to 10 units.

During the exercise test the subjects breathed through a low-resistance valve (Hans Rudolph 2900 device, Kansas City, MO, USA) with a small dead space, and the metabolic and ventilatory variables and parameters were calculated using a specific metabolic analyzer (MMC Horizon System, Sensormedics, Yorba Linda, CA, USA) that provided average values at 15 -s intervals. The individual values of minute ventilation 
$(\dot{\mathrm{V}}), \mathrm{CO}_{2}$ production $\left(\dot{\mathrm{V}}_{2}\right)$ and oxygen uptake $\left(\mathrm{VO}_{2}\right)$ at each power were plotted as a function of time; $\dot{\mathrm{V}}, \dot{\mathrm{V}} \mathrm{CO}_{2}$ and $\dot{\mathrm{VO}}_{2}$ peaks were selected as the highest values reached during the incremental exercise protocol.

In all subjects, anaerobic threshold (in $\dot{\mathrm{VO}}_{2}$ ) was measured when the $\dot{\mathrm{V}}$ and $\dot{\mathrm{VCO}}_{2}$ began to increase non-linearly as compared to $\dot{\mathrm{VO}}_{2}(18)$. This was determined by visual analysis of $\dot{\mathrm{V}}, \dot{\mathrm{VO}}_{2}$ and $\dot{\mathrm{V}} \mathrm{CO}_{2}$ curve responses. Three different observers measured the anaerobic threshold values (in $\mathrm{VO}_{2}$ ) in all exercise tests. Using this procedure, anaerobic threshold (in $\dot{\mathrm{VO}}_{2}$ ) could be measured with a difference of about $2 \%$. In the present study anaerobic threshold was expressed as absolute $(\mathrm{ml} / \mathrm{min})$ and normalized values, i.e., corrected for body weight $(\mathrm{ml}$ $\mathrm{kg}^{-1} \mathrm{~min}^{-1}$ ) and as percentage of peak $\mathrm{VO}_{2}$.

Absolute HR values at anaerobic thresholds and under peak conditions were obtained with an ECG recording system (Funbec, São Paulo, SP, Brazil). The signals were recorded in real time after analog to digital conversion and the R-R intervals (periods expressed in milliseconds between $R-R$ peak waves of ECG signal) were calculated on a beat-to-beat basis using a specific software (19). The HR values are reported as averages at 10 -s intervals.

Protocol II: 24-h Holter electrocardiogram. At least $48 \mathrm{~h}$ after the previous test (Ib), the subjects were submitted to a 24-h Holter recording. The main purpose of this test was to assess the contribution of the autonomic nervous system to the control of HR before and after physical training, by measuring HRV using time and frequency domain methods. ECG signals (leads CM2 and CM5) were recorded using a 24-h Holter tape recorder (Del Mar Avionics, Irvine, CA, USA).

At the beginning of the Holter recording, the volunteers were asked to rest in the supine position for $60 \mathrm{~min}$. After this time, they were instructed on how to proceed through- out the recording period. After a 24-h recording, the volunteers returned to the laboratory to finish the procedure.

The reading and analysis of the ECG recording were done using a Holter Management System (model 750 A Innovator, Del Mar Avionics). A complete automated 24-h report and a visual inspection by the researcher were performed to make sure that the cardiac rhythm was sinusal and that there was no abnormality in atrioventricular electrical conduction. Then, the average HR and $\mathrm{R}-\mathrm{R}$ interval, with the respective standard deviations (time domain), were measured under awake (initial $60 \mathrm{~min}$ - 2:00-3:00 pm) and sleeping conditions (central $6 \mathrm{~h}$ - i.e., without the first and last sleep hours - 0:006:00 am). Following the next step of analysis, the highest stationary sections of R-R intervals on the monitor display were selected for analysis of HRV as a criterion required for correct application of frequency domain analysis, i.e., fast Fourier transform (1).

The data of R-R intervals during a period of resting in the supine position in the awake (2:00-3:00 pm) and sleeping states (0:006:00 am) were analyzed in short-term recordings which included four consecutive nonoverlapping windows of $256 \mathrm{~s}$ each. The data of R-R intervals during the sleep period were analyzed after an initial sleeping time of approximately $160 \mathrm{~min}$ for the young group and $180 \mathrm{~min}$ for the middle-aged group.

The selected time domain parameters studied were the mean R-R interval and the corresponding standard deviation. For frequency domain analysis, the power spectral components are reported at low (0.04 to 0.15 $\mathrm{Hz})$ and high (0.15 to $0.4 \mathrm{~Hz})$ frequencies obtained using the fast Fourier transform in absolute and normalized units. The low/high frequency ratio of absolute power was also measured $(5,20)$. The absolute low and high frequencies are reported as $\mathrm{ms}^{2} / \mathrm{Hz}$ while normalized units were computed by dividing the absolute power of a given low or high 
frequency component by the total power, after subtracting from it the power of the component with a range frequency between 0 and $0.03 \mathrm{~Hz}$, i.e., very low frequency, and multiplying this ratio by $100(1,20)$.

Protocol III: aerobic exercise training. The program was conducted for 3 months on a field track and included stretching for 10 min followed by walking and/or jogging for $40 \mathrm{~min}$, three times a week at a prescribed HR that corresponded to 70 to $85 \%$ of peak HR obtained during a continuous dynamic exercise test performed in a laboratory environment (protocol I-b: functional capacity evaluation). This intensity training range was also based on metabolic profile, since $75 \%$ of peak HR in each volunteer corresponded or was very close to the HR at the anaerobic threshold obtained previously during the oxygen uptake test. Thus, in each training session the subjects were submitted to progressive intensities of exercise (walking and/or jogging) at HR values that were below, equal to and above those related to anaerobic threshold. During the exercise program the subjects used pulse monitors (model Vantage XL, Polar, Port Washington, NY, USA) to ensure they were exercising at the appropriate intensity. The aerobic training intensity was adjusted during a 7-day interval based on rest and exercise HR measured with the above specified pulse monitor, compared to the previous control period (protocol I-b).

\section{Statistical analysis}

The data are reported as medians, quartiles (1st and 3rd) and minimum and maximum values using the Tukey box-plot. Due to nonGaussian distribution and/or inhomogeneity of variance of variable values, nonparametric tests were selected for statistical analysis. Thus, the Mann-Whitney and Wilcoxon nonparametric tests were used for intergroup and intragroup comparisons, respectively, with the level of significance set at $5 \%$.

\section{Results}

The physical characteristics of the young and middle-aged subjects are shown in Table 1. Under control conditions, and after aerobic training, median age, weight and body mass index were higher in the middle-aged than in the young group $(\mathrm{P}<0.05)$; only median height was similar for the two groups. After aerobic training, the intragroup differences in weight and body mass index were not statistically significant.

\section{Exercise conditions}

Responses to dynamic exercise: anaerobic threshold and peak oxygen uptake. Under control conditions, anaerobic threshold and $\mathrm{VO}_{2}$ reported as absolute oxygen uptake and normalized values for body weight were lower $(\mathrm{P}<0.05)$ in middle-aged than in young men (Table 2). Again, under control conditions the values of HR at anaerobic threshold and peak effort, as well as power at anaerobic threshold were significantly lower in middle-aged men than young men (Table 2).

After 3 months of aerobic physical training the absolute and normalized values of $\mathrm{VO}_{2}$ at anaerobic threshold increased significantly $(\mathrm{P}<0.05)$ for both groups; under peak conditions the same occurred for $\mathrm{VO}_{2}$ (absolute) only for the young group and for the power values for both groups. However, under peak conditions the inter- and intragroup

Table 1. Comparison of the anthropometric data for the subjects before (control) and after three months of aerobic physical training (APT).

\begin{tabular}{lcc}
\hline Variable & Young group & Middle-aged group \\
\hline Age (years) & $21^{*}$ & 52 \\
Height $(\mathrm{cm})$ & 174 & 168 \\
Weight $(\mathrm{kg})$, control & $67^{*}$ & 86 \\
Weight $(\mathrm{kg})$, APT & $67^{*}$ & 85 \\
Body mass index $\left(\mathrm{kg} / \mathrm{m}^{2}\right)$, control & $22.9^{*}$ & 28.5 \\
Body mass index $\left(\mathrm{kg} / \mathrm{m}^{2}\right)$, APT & $22.2^{*}$ & 28.2
\end{tabular}

Data are reported as medians. Young group, $\mathrm{N}=10$; middle-aged group, $\mathrm{N}=7$. $* \mathrm{P}<0.05$ for intergroup comparisons (Mann-Whitney test). 
differences in effort perception (Borg's scale) before and after training were not statistically significant (Table 2).

Table 2. Cardiorespiratory variables measured during dynamic exercise before (control, C) and after three months of the aerobic physical training (APT).

\begin{tabular}{|c|c|c|c|c|}
\hline \multirow[t]{2}{*}{ Variable } & \multicolumn{2}{|c|}{ Young group } & \multicolumn{2}{|c|}{ Middle-aged group } \\
\hline & $\mathrm{C}$ & APT & $\mathrm{C}$ & APT \\
\hline$\dot{\mathrm{V}}_{2} \mathrm{AT}\left(\mathrm{ml} \mathrm{kg} \mathrm{kg}^{-1} \mathrm{~min}^{-1}\right)$ & $19 *+$ & $22^{+}$ & $13^{*}$ & 16 \\
\hline $\mathrm{V}_{2}$ peak $\left(\mathrm{ml} \mathrm{kg}{ }^{-1} \mathrm{~min}^{-1}\right)$ & $36^{+}$ & $41^{+}$ & 28 & 27 \\
\hline $\mathrm{V}_{2} \mathrm{AT}(\mathrm{ml} / \mathrm{min})$ & $1292^{*+}$ & $1466^{+}$ & $1186^{*}$ & 1274 \\
\hline $\mathrm{VO}_{2}$ peak (ml/min) & $2630 *+$ & $2800^{+}$ & 2287 & 2410 \\
\hline $\mathrm{HR}(\mathrm{bpm})$ at AT & $134^{+}$ & $142^{+}$ & 115 & 116 \\
\hline HR (bpm) at peak & $191^{+}$ & $188^{+}$ & 158 & 165 \\
\hline Power (watts) at AT & $106^{*+}$ & $130^{+}$ & 77 & 86 \\
\hline Power (watts) at peak & $213^{*}$ & 224 & $171^{*}$ & 183 \\
\hline Borg's scale & 9 & 10 & 8 & 8.5 \\
\hline
\end{tabular}

Data are reported as medians. Young group, $\mathrm{N}=10$; middle-aged group, $\mathrm{N}=7$. AT, anaerobic threshold; $H R$, heart rate.

$* \mathrm{P}<0.05$ for intragroup comparisons (Wilcoxon test).

$+\mathrm{P}<0.05$ for intergroup comparisons (Mann-Whitney test).

Table 3. Comparison of heart rate variability during the resting supine awake condition (2:00-3:00 pm) before (control, C) and after three months of aerobic physical training (APT).

\begin{tabular}{|c|c|c|c|c|}
\hline \multirow[t]{2}{*}{ Variable } & \multicolumn{2}{|c|}{ Young group } & \multicolumn{2}{|c|}{ Middle-aged group } \\
\hline & $\mathrm{C}$ & APT & $\mathrm{C}$ & APT \\
\hline \multicolumn{5}{|l|}{ Time span: 60 min wake } \\
\hline \multicolumn{5}{|l|}{ Time domain indexes } \\
\hline Average heart rate (bpm) & 69* & 60 & $72^{*}$ & 62 \\
\hline Average R-R interval (ms) & $880 *$ & 1003 & $845^{*}$ & 976 \\
\hline Standard deviation average R-R interval (ms) & 85 & 97 & 61 & 64 \\
\hline \multicolumn{5}{|l|}{ Time span: 17 min\# } \\
\hline \multicolumn{5}{|l|}{ Time domain indexes } \\
\hline Average R-R interval (ms) & 869* & 1010 & $833^{*}$ & 1000 \\
\hline Standard deviation average R-R interval (ms) & 83 & 92 & 51 & 55 \\
\hline \multicolumn{5}{|l|}{ Frequency domain indexes } \\
\hline Low frequency $\left(\mathrm{ms}^{2} / \mathrm{Hz}\right)$ & 818* & $1048^{+}$ & 687 & 513 \\
\hline High frequency $\left(\mathrm{ms}^{2} / \mathrm{Hz}\right)$ & 277 & 429 & 265 & 253 \\
\hline Total power $\left(\mathrm{ms}^{2} / \mathrm{Hz}\right)$ & 1821 & 2870 & 2601 & 2942 \\
\hline
\end{tabular}

Heart rate variability was determined using time and frequency domain methods. Data are reported as medians. Young group, $\mathrm{N}=10$; middle-aged group, $\mathrm{N}=7$. \#Four consecutive windows of $256 \mathrm{~s}$ each.

$* \mathrm{P}<0.05$ for intragroup comparisons (Wilcoxon test).

$+\mathrm{P}<0.05$ for intergroup comparisons (Mann-Whitney test).
Holter ECG analysis: R-R interval and its variability in awake and sleeping conditions

Time domain index of HRV: before and after exercise training. In the awake resting supine position the mean R-R interval and its standard deviation did not differ between groups before or after training (Table 3 ). The HR values were lower in the sleeping than in the awake resting supine condition (the inverse for R-R interval) for both groups $(\mathrm{P}<0.05)$. Aerobic physical training induced significant $(\mathrm{P}<0.05)$ bradycardia (increase in average $\mathrm{R}-\mathrm{R}$ interval) of comparable magnitude for both groups studied in the awake resting supine position (Table 3 ).

Table 4 shows that the mean R-R interval and standard deviation throughout the 6-h central sleep were significantly $(\mathrm{P}<0.05)$ higher in the young than in the middle-aged group under control conditions as well as after physical training. The initial time of sleep analysis for short-term time and frequency domains did not differ significantly between groups, being $159 \mathrm{~min}$ for the young group and $183 \mathrm{~min}$ for the middle-aged group. In relation to the effect of physical training on sleep, there were no statistically significant changes in average HR, average R-R intervals or standard deviation of average $\mathrm{R}-\mathrm{R}$ intervals in either group studied.

Frequency domain index of HRV before and after exercise training. Tables 3 and 4 show the HRV in the frequency domain expressed as absolute values in the resting supine position in the awake and sleeping condition, respectively. In the awake control condition no statistical difference could be found in the low frequency, high frequency or total power component between groups, as reported in absolute values. However, after aerobic physical training only the young group presented a significantly higher absolute low frequency power component compared to the control condition $(\mathrm{P}<0.05)$; intergroup analysis showed that in the posttraining condition the young group presented 
a significantly higher absolute low frequency power component than the middle-aged one $(\mathrm{P}<0.05)$.

On the other hand, during the control sleeping condition, the absolute high frequency component, an index of vagal tone, was 7.6 times higher $(\mathrm{P}<0.05)$ in the young than in the middle-aged group (median = 2589 and $342 \mathrm{~ms}^{2} / \mathrm{Hz}$, respectively). This magnitude decreased 2.8 times after training $(\mathrm{P}<0.05)$. The absolute low frequency component during sleep was significantly higher $(\mathrm{P}<0.05)$ in the young than in the middleaged group only in the control condition $\left(1030\right.$ and $\left.357 \mathrm{~ms}^{2} / \mathrm{Hz}\right)$ and not in the aerobic physical training condition (930 and 502 $\mathrm{ms}^{2} / \mathrm{Hz}$ ). Also, the total power was significantly greater $(\mathrm{P}<0.05)$ in the young than in the middle-aged group in the control condition (4862 and $1225 \mathrm{~ms}^{2} / \mathrm{Hz}$, respectively) and in the post-training condition (3152 and $1584 \mathrm{~ms}^{2} / \mathrm{Hz}$, respectively).

The outstanding finding is that the aerobic training did not change any frequency domain component (absolute low and high frequencies) when intragroup comparisons were considered.

When the content of each frequency band was reported as normalized units (normalized high and low frequencies) and as a ratio of absolute values (low/high frequency) the differences were statistically significant only in the sleeping control condition, since normalized low frequency and the low/high frequency ratio were lower and normalized high frequency was higher in the young than in the middle-aged group. After aerobic training the differences were also not statistically significant for inter- or intragroup comparisons (Figures 1 and 2).

\section{Discussion}

In the present study, aerobic exercise training caused an increase in aerobic capacity, i.e., oxygen uptake and transport, indicated by a significant increase in $\mathrm{VO}_{2}$-anaero-
Table 4. Comparison of heart rate variability during sleep (0:00-6:00 am), during control conditions (C) and after three months of aerobic physical training (APT).

\begin{tabular}{|c|c|c|c|c|}
\hline \multirow[t]{2}{*}{ Variable } & \multicolumn{2}{|c|}{ Young group } & \multicolumn{2}{|c|}{ Middle-aged group } \\
\hline & $\mathrm{C}$ & APT & C & APT \\
\hline \multicolumn{5}{|l|}{ Time span: 6 h sleep } \\
\hline \multicolumn{5}{|l|}{ Time domain indexes } \\
\hline Average heart rate (bpm) & $57^{*}$ & $52 *$ & 64 & 64 \\
\hline Average R-R interval (ms) & 1053* & $1165 *$ & 940 & 942 \\
\hline $\begin{array}{l}\text { Standard deviation average } \\
\text { R-R interval (ms) }\end{array}$ & 108* & $124 *$ & 96 & 74 \\
\hline \multicolumn{5}{|l|}{ Time span: $17 \mathrm{~min}^{+}$} \\
\hline $\begin{array}{l}\text { Initial time of data analysis during sleep (min) } \\
\text { Time domain indexes }\end{array}$ & \multicolumn{3}{|c|}{ Time domain indexes } & 245 \\
\hline Average R-R interval (ms) & 1063* & $1120 *$ & 930 & 1035 \\
\hline $\begin{array}{l}\text { Standard deviation average } \\
\text { R-R interval (ms) }\end{array}$ & 70* & 56* & 38 & 47 \\
\hline \multicolumn{5}{|l|}{ Frequency domain indexes } \\
\hline Low frequency $\left(\mathrm{ms}^{2} / \mathrm{Hz}\right)$ & $1030 *$ & 930 & 357 & 502 \\
\hline High frequency $\left(\mathrm{ms}^{2} / \mathrm{Hz}\right)$ & 2589* & $1374 *$ & 342 & 488 \\
\hline Total power $\left(\mathrm{ms}^{2} / \mathrm{Hz}\right)$ & 4862* & $3152 *$ & 1225 & 1584 \\
\hline
\end{tabular}

Heart rate variability was measured using time and frequency domain methods. Data are reported as medians. Young group, $\mathrm{N}=10$; middle-aged group, $\mathrm{N}=7$. ${ }^{+}$Four consecutive windows of $256 \mathrm{~s}$ each.

$* \mathrm{P}<0.05$ for intergroup comparisons (Mann-Whitney test).

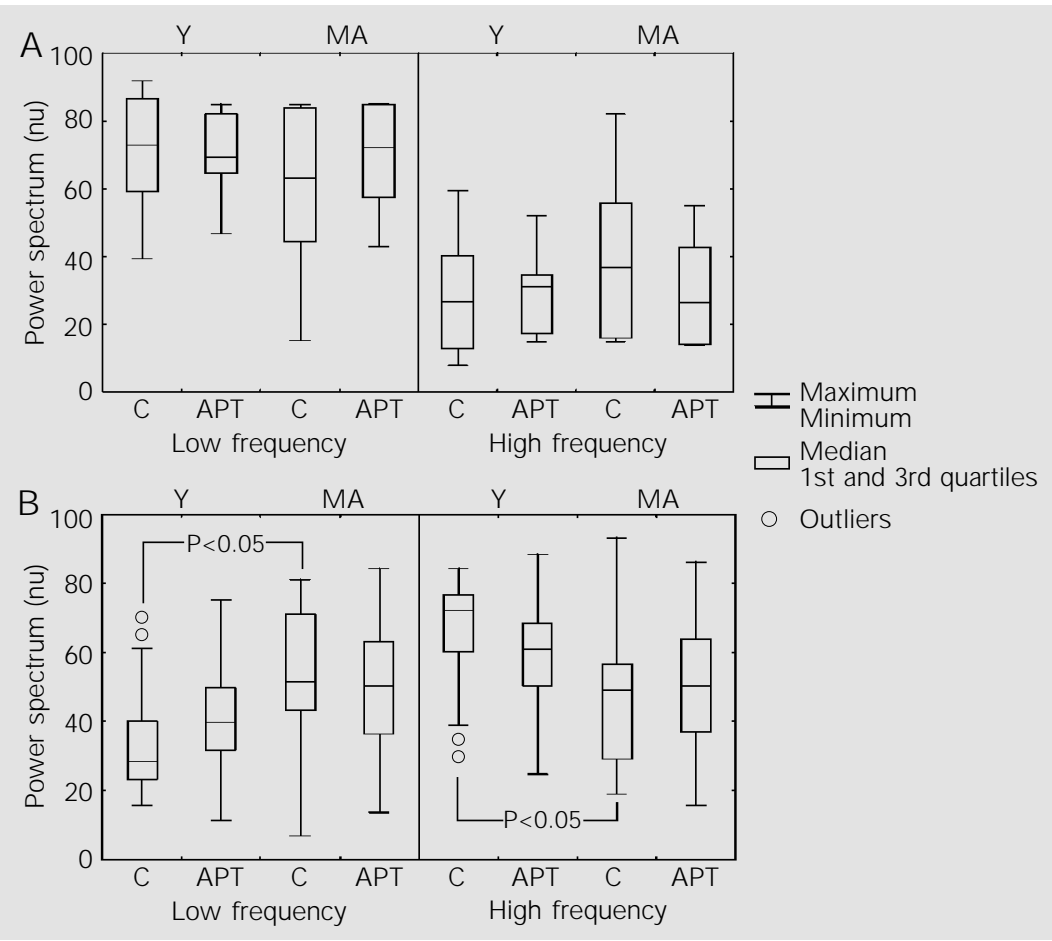

Figure 1. Low and high frequency power spectral components in normalized units (nu), obtained during awake (A) and sleeping (B) conditions, in the young $(\mathrm{Y})$ and middle-aged (MA) groups, during control (C) conditions and after aerobic physical training (APT). Data are reported as box-plots. $\mathrm{P}<0.05$ obtained by the Mann-Whitney test. 
bic threshold in both groups and in peak $\dot{\mathrm{VO}}_{2}$ for the young group only, as well as an increase in power peak for both groups. This increase in aerobic capacity has been documented in other studies $(10,15,16,21,22)$ for both anaerobic threshold and peak $\mathrm{VO}_{2}$. The reasons for the middle-aged group not to increase the peak $\mathrm{VO}_{2}$ may not be due exclusively to the experimental design used, i.e., a longitudinal study with short-time aerobic training. Some possible explanations for this difference are: 1) anthropometric characteristics and the number of subjects studied; 2) type of experimental protocols used, i.e., continuous exercise on a cycloergometer, in relation to the type of exercise training, i.e., walking and jogging on a field track. On the other hand, the perception of effort at peak level, evaluated with Borg's scale (17), was not modified by exercise training despite the increase in aerobic power, suggesting that during exercise the volunteers were pushed to their limits.

In addition, our data showed that the $\dot{\mathrm{V}} \mathrm{O}_{2}$-anaerobic threshold when reported as absolute and normalized $\left(\mathrm{ml} \mathrm{kg}^{-1} \mathrm{~min}^{-1}\right)$ values proved to be more sensitive than peak $\mathrm{VO}_{2}$ in detecting aerobic capacity changes induced by short-term aerobic training in both age groups. It should be emphasized

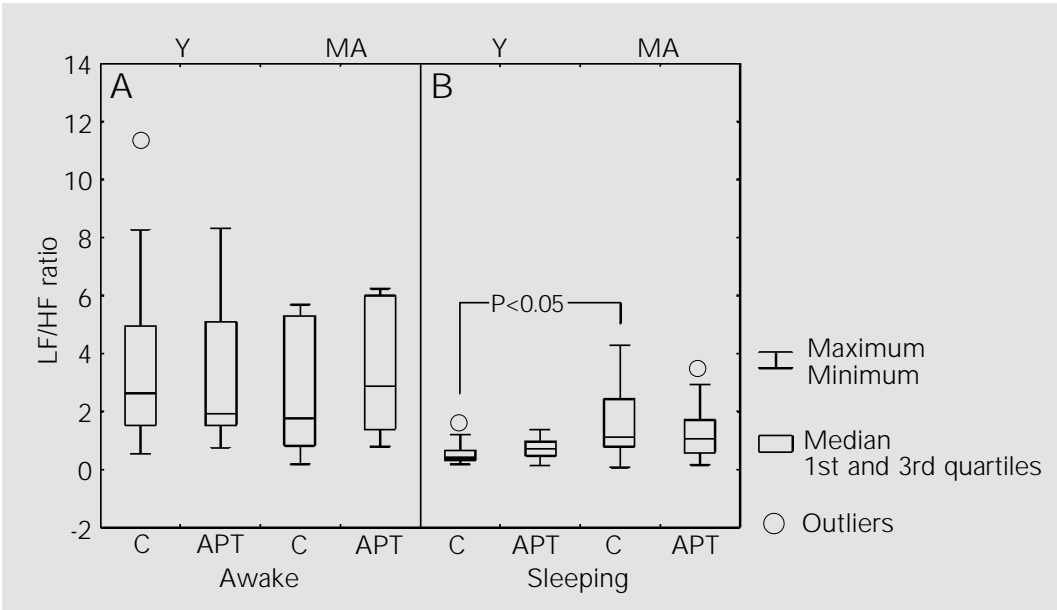

Figure 2. Ratio of the low versus high frequency power spectral components (LF/HF ratio), obtained during awake (A) and sleeping (B) conditions, in the young $(\mathrm{Y})$ and middle-aged (MA) groups, during control (C) conditions and after aerobic physical training (APT). Data are reported as box-plots. $\mathrm{P}<0.05$ obtained by the Mann-Whitney test. that anaerobic threshold has also the advantage of being a parameter measured directly under submaximal testing conditions independently of a required voluntary motivation effort by the subjects, i.e., without the need for vocal reinforcement by the researcher aiming to extend the exercise to the power value needed to obtain the $\dot{\mathrm{VO}}_{2}$ max or peak $\dot{\mathrm{VO}}_{2}$ measurement $(16,18)$.

The HR during the central $6 \mathrm{~h}$ of sleep in both conditions was significantly lower in the young than in the middle-aged group. These data agree with the study of Crasset et al. (23), which documented that the R-R interval did not differ between young and older subjects during awake periods but was higher in the young than in the older subjects during both rapid eye movement (REM) and non-REM sleep. Goldsmith et al. (24) have also reported higher $\mathrm{R}-\mathrm{R}$ interval values during the night in young men as an expression of vagal predominance during sleep.

Under awake control conditions, the intergroup differences of HRV in both the time and frequency domains found in our study were not statistically significant. Nevertheless, during the sleeping situation there were significant differences in parasympathetic modulation between young and middle-aged men. With respect to high frequency power, the higher values found in the young than in middle-aged group support the interpretation that young men exhibit a higher parasympathetic activity during sleep. Our results were similar to those obtained by JensenUrstad et al. (25) who reported a higher high frequency power value in younger than in older subjects during sleep. Crasset et al. (23) also reported that R-R variability was higher in the young subjects than in older volunteers during the awake and sleeping conditions both in the REM and non-REM stages.

As a whole, the above findings of an agedependent difference in R-R intervals and HRV during the sleep condition indicate that the occurrence of vagal predominance in this physiological condition is decreased by aging. 
It should be emphasized that the decrease in vagal tonus in the sinus node has also been reported in pathological conditions such as myocardial infarction, and is associated with an increased risk of new cardiac events and sudden death (8). However, it is not known if the age-dependent decrease in vagal tonus has the same risk effect on the heart of healthy middle-aged men, as documented in the present study.

A limitation of our study was that the 24h ECG recording was not accompanied by polysomnographic sleep recording. Despite this, the established criterion was to analyze intervals with the highest stationary periods of ECG recording during sleep since this condition most likely occurs in the nonREM stages - when there is a shift of cardiac sympathovagal balance, with a corresponding increase in parasympathetic over sympathetic stimulation in the sinus node $(23,26$, 27).

However, it should be emphasized that sleep is a peculiar physiological condition and the mechanisms controlling the high frequency component of R-R variability are unclear. The literature reports that the high frequency component is mediated not only by direct modulation of vagal efferent activity, resulting from baroreceptor responses conveyed to respiratory and blood pressure centers in the central nervous system (28), but also by mechanical effects on the sinus node related to phasic changes in venous return caused by respiratory movement (29).

In spite of these considerations, the reduction of HRV with age in man has been well documented in the literature $(4,7,25)$. However, the mechanisms responsible for this physiological response are unknown. Byrne et al. (4) suggested that age per se, and not the reduction in aerobic capacity or the increase in fat usually associated with the aging process, plays the major role in decreasing HRV in older subjects. Also, it has been shown that the decline in HRV with aging is mainly, but not exclusively, due to a decline in parasympathetic tonus $(23,30)$.

Concerning the effects of aerobic training on HRV, several studies have found HRV modifications in this physiological condition $(7,15,24,31)$. Particularly important is the investigation conducted by Goldsmith et al. (24) who studied and compared 24-h HRV in aerobically trained and untrained healthy young men and observed that parasympathetic activity is substantially greater in trained than in untrained men, during both waking and sleeping hours.

In the present study, although several significant cardiorespiratory adaptations related to oxygen uptake were induced by dynamic training in both groups, they were not accompanied by significant changes in resting HR and HRV (time and frequency domains) during the sleeping condition. However, in the awake condition a resting bradycardia was observed after training in both groups studied, without concomitant changes in time or frequency domain HRV. So, the resting bradycardia observed in this study was not accompanied by an increase of the high frequency component, suggesting a nonsignificant participation of vagal modulation in this adaptive response. In this regard, our results are similar to those of Boutcher and Stein (10) who reported significant increases in both absolute and relative peak $\mathrm{VO}_{2}$ associated with a corresponding decrease in resting HR, without an increase in HRV (analysis in time and frequency domains in a middleaged group (45 years) after aerobic training). In contrast, another study (31) on older awake subjects (67 \pm 5.1 years) observed an increase in HRV in both the time and frequency domain components (very low frequency and low frequency) after 6-month aerobic training or even that exercise training may increase parasympathetic activity throughout the day (24). Moreover, Stein et al. (15), studying the effect of 12-month supervised aerobic training on cardiac autonomic modulation in healthy older adults (66 \pm 4 years), found an increase in total 
HRV and a reduction in nocturnal HR. Also, the same investigators have shown that a sustained increase in HRV lasted over a oneyear period for those who maintained a steady training level.

Thus, the studies discussed above support the fact that in short-term aerobic physical training different mechanisms may be responsible for the resting bradycardia induced by aerobic training. It should be mentioned that this adaptation is a well-documented response reported for both man $(16,25,32)$ and other species (33).

The resting HR is modulated by a balance between sympathetic and parasympathetic tone with a predominance of the latter $(16,34)$. On this basis, some reports state that an increased vagal tonus is the main mechanism for the bradycardia induced by aerobic physical training (35). Goldsmith et al. (24) reported that the bradycardia exhibited by endurance-trained individuals is attributed, at least in part, to greater parasympathetic activity. However, several other studies have failed to demonstrate differences in vagal tone between trained and untrained subjects $(6,32,36,37)$. Yet, others have indicated a decrease in sympathetic activity in the sinus node (38) or both an increase in vagal activity and a decrease in sympathetic activity (39). On the other hand, studies on animals (33) and on humans $(6,32,36,40)$ have suggested that this bradycardia is mainly due to a reduction in intrinsic HR.

Within this context, our data suggest that, at least in men, resting bradycardia induced by short-term aerobic training seems to be mediated by adaptations much more related to intrinsic alterations in the sinus node than to changes in efferent vagal-sympathetic modulation of the sinus node, because the resting bradycardia observed was not accompanied by an increase of the high frequency component and/or a decrease of the low frequency component that would express a higher vagal modulation and/or a low sympathetic modulation of this structure.
Our findings are consistent with previous studies conducted on animals (33) and mainly on humans $(6,32,36,37,40)$ under carefully designed protocols using less invasive or noninvasive procedures associated with better quantitative methods. Nevertheless, Negrão et al. (33) did not exclude the possibility of a decreased resting firing rate of the vagus after training when they observed impairment of vagal function evaluated by reflex bradycardia and electrical vagal stimulation.

The absence of significant changes in HRV associated with an increase in aerobic capacity induced by aerobic training, documented in the present study, may be related to the fact that the experimental design was directed to evaluate the cardiorespiratory adaptation in short-term training. Our data support the results of other studies that documented no HRV change after aerobic exercise training in young (40) and middle-aged men (10).

The results of the present investigation indicate that the vagal predominance during sleep in men is reduced with age. Again, the resting bradycardia induced by short-term aerobic training in both young and middle-aged men is much more related to intrinsic alterations in the sinus node than to changes in efferent vagal-sympathetic modulation. Furthermore, the greater alterations in aerobic capacity than in HRV in both groups may be related to the magnitude of different timedependent responses of each cardiorespiratory variable induced by the training stimulus.

\section{Acknowledgments}

The authors are grateful to Prof. Dr. Eduardo Arantes Nogueira, Disciplina de Cardiologia and to the Departamento de Clínica Médica, Faculdade de Ciências Médicas, Universidade Estadual de Campinas (UNICAMP), where the clinical examination and biochemical tests were performed. We are also indebted to Ricardo Vigatto for help with the preparation of the manuscript. 


\section{References}

1. Task Force of the European Society of Cardiology and the North American Society of Pacing and Electrophysiology (1996). Heart rate variability: standards of measurement, physiological interpretation and clinical use. Circulation, 93: 10431065.

2. Akselrod S, Gordon D, Ubel FA, Shannon DC, Berger AC \& Cohen RJ (1981). Power spectrum analysis of heart rate fluctuation: a quantitative probe of beat-to-beat cardiovascular control. Science, 213: 220222.

3. Skyschally A, Breuer H-WM \& Heusch G (1996). The analysis of heart rate variability does not provide a reliable measurement of cardiac sympathetic activity. Clinical Science, 91 (Suppl): 102-104.

4. Byme EA, Fleg J L, Vaitkevicius PV, Wright J \& Porges SW (1996). Role of aerobic capacity and body mass index in the ageassociated decline in heart rate variability. J oumal of Applied Physiology, 81: 743750.

5. Pagani M, Lombardi F, Guzzetti S, Rimoldi $O$, Furlan R, Pizzinelli $P$, Sandrone G, Malfatto G, Dell'Orto S, Piccaluga E, Turiel M, Baselli G, Cerutti S \& Malliani A (1986). Power spectral analysis of heart rate and arterial pressure variabilities as a marker of sympatho-vagal interaction in man and conscious dog. Circulation Research, 59: 178-193.

6. Martinelli FS, Chacon-Mikahil MPT, Golfetti R, Martins LEB, Lima-Filho EC \& Gallo J r L (2000). Heart rate variability in cyclists and sedentary young men at rest and during head-up tilt. Physiologist, 43: A14-A17, 338 (Abstract).

7. De Meersman RE (1993). Heart rate variability and aerobic fitness. American Heart J ournal, 125: 726-731.

8. Bigger J r JT, Fleiss J L, Steinman RC, Rolnitzky LM, Kleiger RE \& Rottman J N (1992). Frequency domain measures of heart period variability and mortality after myocardial infarction. Circulation, 85: 164171.

9. Sacknoff DM, Gleim GW, Stachenfeld N \& Coplan NL (1994). Effect of athletic training on heart rate variability. American Heart J oumal, 127: 1275-1278.

10. Boutcher SH \& Stein P (1995). Association between heart rate variability and training response in sedentary middleaged men. European J ournal of Applied Physiology, 70: 75-80.

11. Furlan R, Piazza S, Dell'Orto S, Gentile E, Cerutti S, Pagani M \& Malliani A (1993).
Early and late effects of exercise and athletic training on neural mechanisms controlling heart rate. Cardiovascular Research, 27: 482-488.

12. Shin $\mathrm{K}$, Minamitani $\mathrm{H}$, Onishi $\mathrm{S}$, Yamazaki H \& Lee M (1997). Autonomic differences between athletes and nonathletes: spectral analysis approach. Medicine and Science in Sports and Exercise, 29: 14821490.

13. Inbar $O$, Oren $A$, Scheinowitz $M$, Rotstein A, Dlin R \& Casaburi R (1994). Normal cardiopulmonary responses during incremental exercise in 20- to 70-yr-old men. Medicine and Science in Sports and Exercise, 26: 538-546.

14. J ackson AS, Beard EF, Wier LT, Ross RM, Stuteville JE \& Blair SN (1995). Changes in aerobic power of men, ages 25-70 yr. Medicine and Science in Sports and Exercise, 27: 113-120.

15. Stein PK, Ehsani AA, Domitrovich PP, Kleiger RE \& Rottman J N (1999). Effect of exercise training on heart rate variability in healthy older adults. American Heart J ournal, 138 (Part 1): 567-576.

16. Chacon-Mikahil MPT, Forti VAM, Catai AM, Szrajer J S, Golfetti R, Martins LEB, Lima-Filho EC, Wanderley J S, Marin Neto J A, Maciel BC \& Gallo-J unior L (1998). Cardiorespiratory adaptations induced by aerobic training in middle-aged men: the importance of a decrease in sympathetic stimulation for the contribution of dynamic exercise tachycardia. Brazilian J ournal of Medical and Biological Research, 31: 705-712.

17. Borg G (1998). Borg's Perceived Exertion and Pain Scales. Human Kinetics, Champaign, IL, USA.

18. Wasserman $K$, Hansen J E, Sue DY, Whipp BJ \& Casaburi R (1994). Principles of Exercise Testing and Interpretation. 2nd edn. Lea \& Febiger, Philadelphia, PA, USA.

19. Silva $E$, Catai AM, Trevelin LC, Guimarães J O, Silva J $r$ LP, Silva LMP, Oliveira $L$, Milan LA, Martins LEB \& Gallo J unior L (1994). Design of a computerized system to evaluate the cardiac function during dynamic exercise. Physics in Medicine and Biology, 33: 409 (Abstract).

20. Malliani A (1995). Association of heart rate variability components with physiological regulatory mechanisms. In: Malik $M$ \& Camm AJ (Editors), Heart Rate Variability. Futura Publishing Company, Armonk, NY, USA, 173-188.

21. Davis JA, Frank MH, Whipp BJ \& Wasserman K (1979). Anaerobic thresh- old alterations caused by endurance training in middle-aged men. J ournal of Applied Physiology: Respiratory, Environmental and Exercise Physiology, 46: 10391046.

22. Gregoire J, Tuck S, Yamamoto $Y \&$ Hughson RL (1996). Heart rate variability at rest and exercise: influence of age, gender, and physical training. Canadian J ournal of Applied Physiology, 21: 455470.

23. Crasset V, Mezzetti $S$, Antoine $M$, Linkowski $P$, Degaute JP \& van de Bome $P$ (2001). Effects of aging and cardiac denervation on heart rate variability during sleep. Circulation, 103: 84-88.

24. Goldsmith RL, Bigger J r J T, Steinman RC \& Fleiss JL (1992). Comparison of 24hour parasympathetic activity in endurance-trained and untrained young men. J ournal of the American College of Cardiology, 20: 552-558.

25. J ensen-Urstad K, Storck N, Bouvier $F$, Ericson M, Lindblad LE \& J ensen-Urstad M (1997). Heart rate variability in healthy subjects is related to age and gender. Acta Physiologica Scandinavica, 160: 235241.

26. Scholz UJ , Bianchi AM, Cenutti S \& Kubicki S (1997). Vegetative background of sleep: spectral analysis of the heart rate variability. Physiology and Behavior, 62: 10371043.

27. Elsenbruch S, Harnish MJ \& Orr WC (1999). Heart rate variability during waking and sleep in healthy males and females. Sleep, 22: 1067-1071.

28. Piepoli $M$, Sleight $P$, Leuzzi $S$, Valle $F$, Spadacini G, Passino C, J ohnston J \& Bernardi $L$ (1997). Origin of respiratory sinus arrhythmia in conscious humans. An important role for arterial carotid baroreceptors. Circulation, 95: 1813-1821.

29. Bernardi L, Keller F, Sanders M, Reddy PS, Griffith B, Meno F \& Pinsky MR (1989). Respiratory sinus arrhythmia in the denervated human heart. J ournal of Applied Physiology, 67: 1447-1455.

30. Shannon DC, Carley DW \& Benson H (1987). Aging of modulation of heart rate. American J oumal of Physiology, 253 (Part 2): H874-H877.

31. Schuit AJ, van Amelsvoort LGPM, Verheij TC, Rijneke RD, Maan AC, Swenne CA \& Schouten EG (1999). Exercise training and heart rate variability in older people. Medicine and Science in Sports and Exercise, 31: 816-821.

32. Katona PG, McLean M, Dighton DH \& 
Guz A (1982). Sympathetic and parasympathetic cardiac control in athletes and nonathletes at rest. Journal of Applied Physiology: Respiratory, Environmental and Exercise Physiology, 52: 1652-1657.

33. Negrão CE, Moreira ED, Santos MCLM, Farah VMA \& Krieger EM (1992). Vagal function impairment after exercise training. J oumal of Applied Physiology: Respiratory and Environmental Exercise, 72: 1749-1753.

34. Lakatta EG (1995). Cardiovascular system. In: Masoro EJ (Editor), Handbook of Physiology. A Critical Comprehensive Presentation of Physiological Knowledge and Concepts. Oxford University Press, New York, NY, USA, 413-474.
35. Smith ML, Hudson DL, Graitzer HM \& Raven PB (1989). Exercise training bradycardia: the role of autonomic balance. Medicine and Science in Sports and Exercise, 21: 40-44.

36. Maciel BC, Gallo J unior L, Marin Neto J A, Lima Filho EC, Terra Filho J \& Manço J C (1985). Parasympathetic contribution to bradycardia induced by endurance training in man. Cardiovascular Research, 19: 642-648.

37. Perrault $H$, Gagnon $M C$, J ohnson $D$, Mokrane A \& Nadeau RA (1996). An enhanced vagal influence does not explain training-induced bradycardia. Physiologist, 39: A20 (Abstract).

38. Williams RS, Eden RS, Moll ME, Lester
RM \& Wallace AG (1981). Autonomic mechanisms of training bradycardia: betaadrenergic receptors in humans. J ournal of Applied Physiology: Respiratory, Environmental and Exercise Physiology, 51: 1232-1237.

39. Ekblom B, Kilbom A \& Soltysiak J (1973). Physical training, bradycardia, and autonomic nervous system. Scandinavian J oumal of Clinical and Laboratory Investigation, 32: 251-256.

40. Reiling MJ \& Seals DR (1988). Respiratory sinus arrhythmia and carotid baroreflex control of heart rate in endurance athletes and untrained controls. Clinical Physiology, 8: 511-519. 